\title{
Methodology and Rationale for Ophthalmic Examinations in the Seventh and Eighth Korea National Health and Nutrition Examination Surveys (2017-2021)
}

\author{
Su Jeong Song ${ }^{1}$, Kyung Seek Choi ${ }^{2}$, Jong Chul Han ${ }^{3}$, Donghyun Jee ${ }^{4}$, Jin Wook Jeoung ${ }^{5}$, Young Joon Jo ${ }^{6}$, \\ Jae Yong Kim , Ko Eun Kim ${ }^{8}$, Seong Taeck Kim ${ }^{9}$, Ji Woong Lee ${ }^{10}$, Tae Eun Lee ${ }^{11}$, Dong Hui Lim³ \\ Chan Yun Kim ${ }^{12}$, Hyun Woong Kim ${ }^{13}$, Sang Woo Park ${ }^{14}$, Ki Ho Park ${ }^{5}$, Sang Jun Park ${ }^{15}$, Min Sagong ${ }^{16}$, \\ Jae Pil Shin ${ }^{17}$, Chungkwon Yoo ${ }^{18}$, Yoonjung Kim ${ }^{19}$, Kyungwon $\mathrm{Oh}^{19}$, Kyu Hyung Park ${ }^{15}$ \\ ${ }^{1}$ Department of Ophthalmology, Kangbuk Samsung Hospital, Sungkyunkwan University School of Medicine, Seoul, Korea \\ ${ }^{2}$ Department of Ophthalmology, Soonchunhyang University Seoul Hospital, Soonchunhyang University College of Medicine, Seoul, \\ Korea \\ ${ }^{3}$ Department of Ophthalmology, Samsung Medical Center, Sungkyunkwan University School of Medicine, Seoul, Korea \\ ${ }^{4}$ Department of Ophthalmology, St. Vincent's Hospital, The Catholic University of Korea College of Medicine, Suwon, Korea \\ ${ }^{5}$ Department of Ophthalmology, Seoul National University Hospital, Seoul National University College of Medicine, Seoul, Korea \\ ${ }^{6}$ Department of Ophthalmology, Chungnam National University Hospital, Daejon, Korea \\ ${ }^{7}$ Department of Ophthalmology, Asan Medical Center, University of Ulsan College of Medicine, Seoul, Korea \\ ${ }^{8}$ Department of Ophthalmology, Ajou University Medical Center, Ajou University School of Medicine, Suwon, Korea \\ ${ }^{9}$ Department of Ophthalmology, Chosun University School of Medicine, Gwangju, Korea \\ ${ }^{10}$ Department of Ophthalmology, Pusan National University School of Medicine, Busan, Korea \\ ${ }^{11}$ Department of Ophthalmology, Jeonbuk National University Hospital, Jeonbuk National University Medical School, Jeonju, Korea \\ ${ }^{12}$ Institute of Vision Research, Department of Ophthalmology, Yonsei University College of Medicine, Seoul, Korea \\ ${ }^{13}$ Department of Ophthalmology, Haeundae Paik Hospital, Inje University College of Medicine, Busan, Korea \\ ${ }^{14}$ Department of Ophthalmology, Chonnam National University Medical School, Gwangju, Korea \\ ${ }^{15}$ Department of Ophthalmology, Seoul National University Bundang Hospital, Seoul National University College of Medicine, \\ Seongnam, Korea \\ ${ }^{16}$ Department of Ophthalmology, Yeungnam University Hospital, Yeungnam University College of Medicine, Daegu, Korea \\ ${ }^{17}$ Department of Ophthalmology, Kyungpook National University Hospital, Kyungpook National University School of Medicine, \\ Daegu, Korea \\ ${ }^{18}$ Department of Ophthalmology, Korea University College of Medicine, Seoul, Korea \\ ${ }^{19}$ Division of Health and Nutrition Survey and Analysis, Korea Disease Control and Prevention Agency, Cheongju, Korea
}

This report provides a detailed description of the methodology for ophthalmic examinations according to the Korea National Health and Nutrition Examination Survey (KNHANES) VII and VIII (from 2017 to 2021). The KNHANES is a nationwide survey which has been performed since 1998 in representatives of whole Korean population. During the KNHANES VII and VIII, in addition to the ophthalmic questionnaire, intraocular pressure measurement, visual field test, auto refractometry, axial length

Received: January 28, 2021 Final revision: June 7, 2021 Accepted: June 9, 2021

Corresponding Author: Kyu Hyung Park, MD, PhD. Department of Ophthalmology, Seoul National University Bundang Hospital, Seoul National University College of Medicine, 82 Gumi-ro 173beon-gil, Bundang-gu, Seongnam 13620, Korea. Tel: 82-31-787-7373, Fax: 82-31-787-4057, E-mail: jiani4@, snu.ac.kr 
and optical coherence tomography measurements were included. This new survey will provide not only provide normative and pathologic ophthalmic data including intraocular pressure, refractive error, axial length, visual field and precise measurement of anterior segment, macula and optic nerve with optical coherence tomography, but also a more accurate diagnosis for major adult blindness diseases, including age-related macular degeneration, diabetic retinopathy, glaucoma, and other ocular diseases, for the national Korean population.

Key Words: Korea National Health and Nutrition Examination Survey, Methods, Ophthalmology

\section{Introduction}

For more than two decades, the Korea National Health and Nutrition Examination Survey (KNHANES) has been conducted to evaluate the health and nutritional status of the South Korean population [1-5]. Although the KNHANES provides valuable population-based health information, it did not include vision or ophthalmic examination questions until 2008. To meet the growing need for comprehensive population-based ophthalmic data, detailed ophthalmic examinations performed by ophthalmologists were included in the KNHANES starting in 2008 [6,7]. Ophthalmic examinations were included in the survey in order to investigate the prevalence and risk factors of common eye diseases, such as visual impairment, refractive errors, strabismus, blepharoptosis, cataract, pterygium, diabetic retinopathy (DR), age-related macular degeneration (AMD), glaucoma, dry eye disease, and color vision deficiency.

Since then, there have been a tremendous number of reports provided to the Korean public and worldwide [8-13]. The importance of a national population study cannot be overrated. The updated and reliable national data regarding the epidemiology of major eye diseases is the most important step to establish public health policies to fight against blindness and reduce the socioeconomic burden. Nevertheless, unmet needs have still existed since the sixth KNHANES ophthalmic survey. An accurate diagnosis of glaucoma or subtype classification of AMD was not feasible from the previous KNHANES ophthalmic survey due to the absence of objective test results, such as optical coherence tomography (OCT), which is now crucial for the diagnosis of macular diseases and glaucoma [14,15].

After the success of the previous KNHANES ophthalmic survey and to further embrace previous achievements, the Korean Ophthalmologic Society and Korea Disease Control and Prevention Agency (KDCA, formerly Korea Centers for Disease Control and Prevention) planned another ambitious ophthalmic examination and survey which started in end of KNHANES VII and throughout KNHANES VIII (2017-2021). In this survey, the intraocular lens (IOL) master and anterior and posterior OCT for precise and objective measurement of ophthalmic structures was newly adopted. This information will provide additional yet crucial objective nationwide population-based data regarding AMD and glaucoma prevalence which is based on OCT. Ultimately, these data will help to establish the most accurate diagnosis for major eye diseases in the Korean population.

\section{KNHANES and Ophthalmic Survey Overview}

The KDCA conducted the KNHANES series (I, II, and III) in 1998, 2001, and 2005, respectively, to examine the general health and nutritional status of Koreans. After completion of the KNHANES IV (2007-2009), the survey became an annual project. The study methodology involved stratified multistage cluster-sampling to prevent subject omission or overlap. The rolling-sampling method ensured the representativeness of each annual survey of the overall Korean population, which allowed results to be merged between surveys [6]. More detailed information about the sampling method is available in a previous paper [6]. From July 2008, ophthalmologic interviews and examinations have been conducted (Table 1,2). All examinations and health interviews were conducted by trained teams in mobile units that traveled to each survey location, while nutrition surveys were performed in individual 
Table 1. List of ophthalmic questionnaires in the KNHANES from 2008 to 2021

\begin{tabular}{|c|c|c|c|c|c|c|c|c|c|}
\hline & 2008 & 2009 & 2010 & 2011 & 2012 & $2013-2015$ & 2016 & $2017-2018$ & 2019-2021 \\
\hline History of ocular exam & Yes & Yes & Yes & Yes & Yes & No & Yes & No & No \\
\hline History of recent ocular exam & Yes & Yes & Yes & Yes & Yes & Yes & Yes & Yes & Yes \\
\hline Family history of eye disease & Yes & Yes & Yes & Yes & Yes & Yes & Yes & No & No \\
\hline History of cold extremities \& migraine & Yes & Yes & Yes & Yes & Yes & No & No & No & No \\
\hline Ultraviolet exposure time & Yes & Yes & Yes & Yes & Yes & No & No & No & No \\
\hline History of ophthalmic surgery & No & No & Yes & Yes & Yes & Yes & Yes & Yes & Yes \\
\hline Dry eye & No & No & Yes & Yes & Yes & No & No & No & No \\
\hline Cataract & No & No & Yes & Yes & Yes & Yes & Yes & Yes & Yes \\
\hline Age-related macular degeneration & No & No & Yes & Yes & Yes & Yes & Yes & Yes & Yes \\
\hline Glaucoma & No & No & Yes & Yes & Yes & Yes & Yes & Yes & Yes \\
\hline Diabetic retinopathy & No & No & No & No & No & No & No & No & Yes \\
\hline Contact lens wear & No & No & No & No & No & No & No & No & Yes \\
\hline Near-work duration & No & No & No & No & No & Yes & Yes & Yes & Yes \\
\hline History of parental myopia & No & No & No & No & No & Yes & Yes & No & No \\
\hline
\end{tabular}

KNHANES IV, 2007-2009; V, 2010-2012; VI, 2013-2015; VII, 2016-2018; VIII, 2019-2021.

KNHANES $=$ Korea National Health and Nutrition Examination Survey.

Table 2. List of ophthalmic examinations in the KNHANES from 2008 to 2021

\begin{tabular}{|c|c|c|c|c|c|c|c|c|c|}
\hline & 2008 & 2009 & 2010 & 2011 & 2012 & $2013-2015$ & 2016 & $2017-2018$ & 2019-2021 \\
\hline Visual acuity & Yes & Yes & Yes & Yes & Yes & Yes & Yes & Yes & Yes \\
\hline Autorefraction & Yes & Yes & Yes & Yes & Yes & Yes & Yes & Yes & Yes \\
\hline Strabismus test & Yes & Yes & Yes & Yes & No & No & No & No & No \\
\hline Blepharoptosis & Yes & Yes & Yes & Yes & Yes & No & No & No & No \\
\hline Slit lamp biomicroscopy & Yes & Yes & Yes & Yes & Yes & No & No & No & No \\
\hline Fundus photography & Yes & Yes & Yes & Yes & Yes & No & No & Yes & Yes \\
\hline Color vision & No & No & No & No & No & Yes & No & No & No \\
\hline Axial length & No & No & No & No & No & No & No & Yes & Yes \\
\hline Optical coherence tomography & No & No & No & No & No & No & No & Yes & Yes \\
\hline Intraocular pressure/visual field & Yes & Yes & Yes & Yes & Yes & No & No & Yes & Yes \\
\hline
\end{tabular}

KNHANES IV, 2007-2009; V, 2010-2012; VI, 2013-2015; VII, 2016-2018; VIII, 2019-2021.

KNHANES $=$ Korea National Health and Nutrition Examination Survey.

households. These mobile units provided a standardized environment and standardized equipment. For quality control of ophthalmological exams and the survey, the KDCA and the Korean Ophthalmological Society conducted team education and training programs twice a year. The educational information included the overall purpose of epidemiological studies, cautions, machine operation, and the diagnosis and classification of major eye disorders to be in- vestigated. The quality of the ophthalmic survey was verified by the Epidemiologic Survey Committee of the Korean Ophthalmological Society. In the KNHANES IV-V (2008-2012), a total of 37,982 (17,040 male and 20,942 female) participants received an eye examination. Ophthalmic examinations were temporarily stopped in $\mathrm{KN}$ HANES VI (2013-2015) and restarted in the middle of KNHANES VII (2016-2018). It will be continued in KN- 
HANES VIII (2019-2021). In this survey from 2017 to 2021, all ophthalmic questionnaires and examinations were conducted not by ophthalmologists but by trained nurses. From 2017 to 2020, only adult participants who were older than 40 years were included because the survey was focused on more detailed information about the diseases causing elderly blindness, including AMD, DR, and glaucoma. In 2021, 10- to 59-year-old participants were included to evaluate the prevalence of DR and glaucoma in adolescents and young adults. Previously, 3 year or older

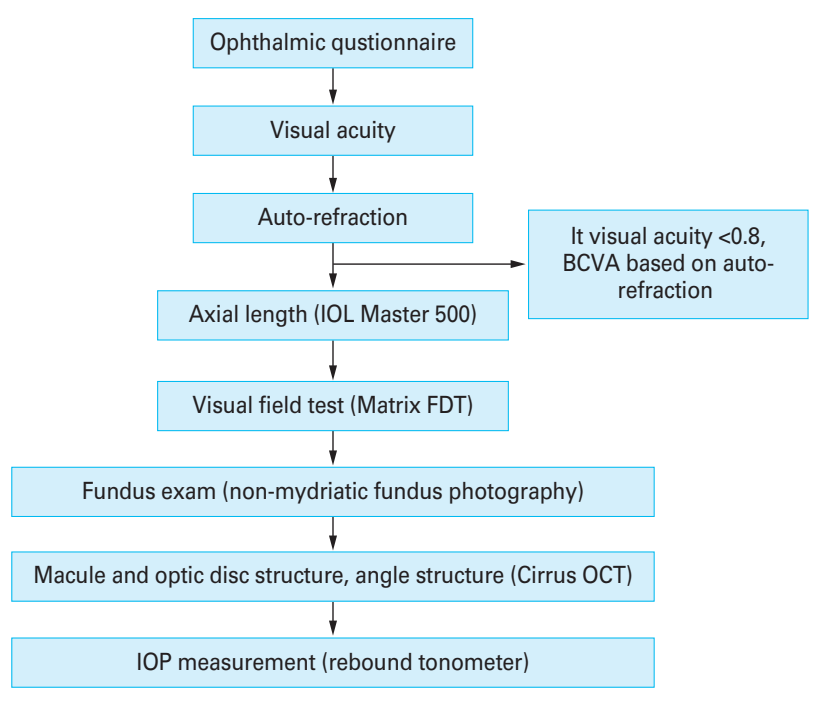

Fig. 1. Representative flow chart of the ophthalmic examination for the Korea National Health and Nutrition Examination Survey VII and VIII. BCVA = best-corrected visual acuity; IOL = intraocular lens; FDT = frequency doubling technology; OCT = optical coherence tomography; IOP = intraocular pressure. participants were included in the 2008 to 2012 surveys, 19to 49-year-old participants were included in 2013 to 2015 , and 5- to 18-year-old participants were included in 2016. From 2017 to 2021, ophthalmic questionnaires were performed that including the history of recent ocular examinations, diagnostic history of ocular diseases, history of ophthalmic surgery, current ocular medication, near-work duration, experience with contact lens wear, and anophthalmia or eyeball removal (Table 1). The ophthalmic examinations, included best-corrected visual acuity, autorefraction, intraocular pressure (IOP) measurement, visual field test (Humphrey frequency doubling technology [FDT] Perimeter; Carl Zeiss Meditec, Dublin, CA, USA), axial length measurement (IOL Master 500, Humphrey FDT Perimeter, Carl Zeiss Meditec), non-mydriatic fundus photography, and OCT (CIRRUS HD-OCT 500, Carl Zeiss Meditec) (Table 2). A dilated fundus examination was not performed to prevent unexpected accidents like falling down when elderly people got on and off the mobile examination unit in this survey. Fig. 1 represents a simplified test flow for the ophthalmological examination.

\section{Ophthalmic Examination Methods}

The details of the individual test methodology for each disease are described in the order they were performed. The study list and their output index are shown in Table 3.

Table 3. List of ophthalmic examinations and output indexes

\begin{tabular}{lll}
\hline Examination method & \multicolumn{1}{c}{ Measurement category } & Output index \\
\hline Visual acuity & Visual acuity & Visual acuity \\
Autorefraction & Refraction & Refractive error \\
IOL master & Axial length & Axial myopia \\
Visual field (FDT) & Visual field & Glaucoma \\
Fundus phototography & Shape of post pole structure \& optic disc & AMD, diabetic retinopathy, CSME, glaucoma, other \\
& abnormality & macular pathology (ERM, MH, RVO, etc.) \\
OCT & Macule \& optic disc structure & Glaucoma, AMD, CSME, other macular pathology \\
& & (ERM, MH, RVO, etc.) \\
IOP measurement & IOP & Glaucoma, ocular hypertension
\end{tabular}

$\overline{\mathrm{IOL}}=$ intraocular lens; FDT = frequency doubling technology; $\mathrm{AMD}=$ age-related macular degeneration; $\mathrm{CSME}=$ clinically significant macular edema; $\mathrm{ERM}=$ epiretinal membrane; $\mathrm{MH}$ = macular hole; $\mathrm{RVO}=$ retinal vein occlusion; $\mathrm{OCT}=$ optical coherence tomography; $\mathrm{IOP}=$ intra ocular pressure. 


\section{Visual acuity and autorefraction}

Uncorrected visual acuity (VA) and/or best-corrected distance VA were measured at a distance of 4 meters using an international standard vision chart based on the Snellen scale (Jin's vision chart; JV Institute, Seoul, Korea). Participant VA was measured in each eye, with the right side followed by the left side. Each participant was asked to read the numbers in the 0.2 line of the VA chart and to proceed to the next line if he or she correctly read at least three of the five letters. Participant VA was defined as the line with the smallest numbers in which the participant accurately read more than three characters. For those participants who had a VA score lower than 0.8 , corrected VA was measured using autorefraction. Automated refraction was performed using an autorefractive keratometer (KR8800; Topcon, Tokyo, Japan), followed by VA retesting using a pinhole in patients with Snellen VA lower than 0.8 . Spherical equivalent refractive error was calculated as sphere $+1 / 2$ cylinder.

\section{Visual field test}

Automated visual field examination was performed on all subjects with FDT, N30-5 screening, Humphrey Matrix (Carl Zeiss Meditec) using standard procedures. Only reliable visual fields, defined as $\leq 1$ fixation losses and false-positive responses, were included. An abnormal visual field was defined as the presence of at least one location of reduced sensitivity.

\section{IOL master}

Due to screening purposes, an objective yet fast and noncontact method was needed for biometry measurements. Therefore, anterior segment dimensions were evaluated with optical biometry (IOL Master 500, Carl Zeiss Meditec). Only axial length measurement was measured in this survey. Anterior chamber depth was not included because it is measured with anterior segment OCT.

\section{Fundus photography}

Fundus photography (VISUCAM 224, Carl Zeiss Meditec) was performed to obtain an image of the fundus of the eye at a field angle of $45^{\circ}$, in eyes with small pupils, the field angle was $30^{\circ}$. Digital images were captured under physiological mydriasis in all participants. For each participant, one fundus image including the macula and optic disc was obtained per eye. Red free photography was also obtained using digital transformation of original color fundus photography. None of the participants had dilated fundus photography taken in this survey.

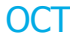

Each subject underwent comprehensive posterior and anterior segment imaging with Cirrus high-definition (HD) OCT (Cirrus HD-OCT 500, Carl Zeiss Meditec). We obtained posterior segment imaging using the built-in program including the Macular Cube $512 \times 128$ images, two HD 5 Line Raster, and Optic Disc Cube $200 \times 200$ protocols, and anterior segment imaging using the built-in 15.5mm Wide ChamberView protocol.

The Macular Cube $512 \times 128$ consisted of 65,536 A-scans in a $6 \times 6 \times 2$-mm cube centered on the fovea. The horizontal and vertical raster scans were obtained by averaging the HD 5 Line Raster protocol. The ganglion cell analysis algorithm reported the macular ganglion cell/inner plexiform layer thickness of six wedge-shaped sectors in a $4.8 \times$ 4.0-mm ellipse, excluding an inner elliptical annulus $1.2 \times$ $1.0 \mathrm{~mm}$ in diameter. The central macular thickness and subfoveal choroidal thickness were independently measured by two board-certified ophthalmologists using the Cirrus HD-OCT Review Software (Carl Zeiss Meditec) in each eye. The central macular thickness was defined as the distance between the internal limiting membrane and the surface of retinal pigment epithelium at the fovea. The subfoveal choroidal thickness was defined as the distance between the Bruch's membrane and the choroidoscleral interface at the macular region. The Optic Disc Cube $200 \times$ 200 consisted of 40,000 A-scans in a $6 \times 6 \times 2$-mm cube centered on the optic nerve head. The Cirrus HD OCT algorithm reported the peripapillary retinal nerve fiber layer thickness and optic nerve head parameters by analyzing the Optic Disc Cube $200 \times 200$ images. The 15.5 -mm Wide ChamberView provided the scan aligned on the corneal apex using the typical vertex reflection on the corneal apex. The central corneal thickness (CCT) and anterior chamber depth (ACD) were independently measured by two board-certified ophthalmologists using the built-in CCT/ACD measuring tool in the Cirrus HD-OCT Review 
Software in each eye. The CCT was defined as the distance from the corneal epithelium to the endothelium at the corneal center and the ACD was defined as the distance from the corneal endothelium to the anterior surface of the lens which was perpendicular to the iris plane.

\section{Rebound tonometry}

All subjects underwent IOP measurements in a sitting position with rebound tonometry (Icare PRO; Icare Finland Oy, Helsinki, Finland). To take IOP measurements with the Icare PRO, the subject was asked to look straight ahead, and the probe was placed within 3 to $7 \mathrm{~mm}$ of the cornea and perpendicular to the central cornea. Six readings were taken and an average value was generated automatically. Below this IOP result, measurement reliability was indicated. An inter-measurement numerical deviation within the normal limits was shown on a green background; The green color means that there has not been too much deviation in the measurements and the measurement is successful. The yellow color indicates that during the six probe shots, there has been some deviation between the results of the probe shots. Red light indicates different types of errors or that the measurement has failed for some reason.

\section{Data Processing and Management}

In each of the four mobile examination units, a picture archiving and communication system (Forum, Carl Zeiss Meditec) was built to manage the scheduled studies and to collect and archive the images and examination results from FDT, optical biometry, nonstereo fundus photography, and OCT. Before the scheduled examination, a work list for each participant in the picture archiving and communication system system was established, which enabled convenient schedule management and allowed automated, reliable, error-free transfer of participants' ID, and demographic information to each study modality.

\section{Diagnostic Criteria of Target Diseases}

AMD

Patients were considered to have early AMD if they demonstrated the presence of soft indistinct drusen or reticular drusen or the presence of hard or soft distinct drusen with pigmentary abnormalities (increased pigmentation or hypopigmentation of the retinal pigment epithelium) in the absence of signs of late AMD [16]. Late AMD included the signs of wet AMD or geographic atrophy. Wet AMD was defined as retinal pigment epithelial detachment or serous detachment of the sensory retina, subretinal or subretinal pigment epithelium hemorrhages, and subretinal fibrous scars. Geographic atrophy was defined as a circular discrete area (175 microns in diameter or greater) of retinal depigmentation with visible choroidal vessels, in the absence of signs of wet AMD [16]. In addition to the fundus findings, OCT findings for dry and wet AMD were added to this survey. Although definite wet AMD findings were not observed with fundus photography, eyes with early AMD features with definite OCT findings, including pigment epithelial detachment or subretinal fluid, were considered to have wet AMD.

DR

DR was identified in the presence of any characteristic lesion determined by the Early Treatment for Diabetic Retinopathy Study (ETDRS) severity scale; i.e., microaneurysms, hemorrhages, hard exudates, cotton wool spots, intraretinal microvascular abnormalities, venous beading, and retinal new vessels. Clinically significant macular edema (CSME) was defined by the ETDRS definition as follows [17].

Thickening of the retina at or within 500 microns of the center of the macula, or hard exudates at or within $500 \mathrm{mi}$ crons of the center of the macula, or if associated with thickening of the adjacent retina, a zone or zones of retinal thickening one disc size or larger, any part that was within one disc diameter of the center of the macula [17]. As for the definition of AMD, both fundus photography and macular thickness measurement findings with OCT using the EDTRS 6-mm circle were used for confirmation of cystoid macular edema. Eyes not having characteristic features of CSME, but with fovea thickening more than 300 microns, 
as documented by OCT within the ETDRS grid, were also considered to have cystoid macular edema.

\section{Open-angle glaucoma, glaucoma suspect, ocular hy- pertension, and angle closure glaucoma}

Participants were considered to have open-angle glaucoma based on the findings of nonmydriatic fundus photography and visual field examination according to International Society of Geographical and Epidemiological Ophthalmology criteria $[18,19]$. The specific diagnostic criteria are as follows: (1) open angle on anterior segment OCT, (2) a reliable visual field defect consistent with glaucoma (no fixation error and false-positive error $\leq 1$ and the presence of at least one location of reduced sensitivity that corresponds to the optic disc or retinal nerve fiber layer abnormality), (3) at least one of the glaucomatous structural changes such as neuroretinal rim notching or thinning, a vertical cup-to-disc ratio $\geq 0.7$, asymmetrical cup-to-disc ratio $\geq 0.3$ and a retinal nerve fiber layer defect. In the case of no reliable visual field test result (no visual field test, visual field test with fixation error or false-positive error $\geq 2$ ), the diagnostic criteria were as follows: (1) open angle on anterior segment OCT, (2) at least one of the glaucomatous structural changes such as neuroretinal rim thinning or notching with a cup-to-disc ratio $\geq 0.9$ or with a corresponding retinal nerve fiber layer defect, asymmetric cupto-disc ratio $\geq 0.3$. If there were no available visual field test results and fundus images, only VA, IOP and a history of glaucoma were used in glaucoma diagnosis. The diagnostic criteria were as follows: (1) corrected VA $\leq 10$ / 200 and IOP $>97.5$ th percentile, (2) corrected VA $\leq 10 / 200$ and a history of glaucoma (glaucoma surgery, medication, and diagnosis). Cirrus HD-OCT was used as the reference and it helped to confirm the definition of glaucoma when it showed the following: (1) a yellow/red colored area in the clock-hour or quadrant map, (2) yellow/red pixels $(>10)$ in the deviation map, or (3) a blue/black area in the thickness map. Circumpapillary and ganglion cell-inner plexiform layer scans were used in the reference of the diagnosis. When the OCT had bad quality, such as signal strength $<6$, inaccurate RNFL segmentation or poor disc margin delineation, the OCT was not used as the reference. When the diagnosis from OCT was not consistent with the diagnosis from fundus photography, the final diagnosis was confirmed by consensus.
Eyes were classified as having suspect glaucoma if they had at least one of the following glaucomatous structural changes such as neuroretinal rim notching or thinning, a vertical cup-to-disc ratio $\geq 0.7$, asymmetrical cup-to-disc ratio $\geq 0.3$ and a retinal nerve fiber layer defect, and open angles on anterior segment OCT with the absence of a glaucomatous defect in the visual field test. In the present study, IOP $<97.5$ th percentile $(23 \mathrm{mmHg}$ ) measured with an Icare tonometer was regarded as "normal". Thus, eyes were classified as having ocular hypertension if IOP $\geq 23 \mathrm{mmHg}$ with open angles without evidence of glaucomatous structural changes and visual field defects. Eyes were classified as having angle closure glaucoma if they had glaucomatous optic neuropathy based on International Society of Geographical and Epidemiological Ophthalmology criteria and glaucomatous visual field defects and closed angles on anterior segment OCT $[18,19]$.

\section{Interpretation of Ophthalmic Examinations}

Experienced retinal specialists selected by the Korean Retina Society who had expertise in grading of DR and AMD performed diagnoses of DR, AMD and other retinal diseases. All the retinal images were read twice by an independent grader. If there was a disagreement in the primary diagnosis, an independent senior reading committee from the Korean Retina Society made the final diagnosis of the retinal diseases.

Experienced glaucoma specialists selected by the Korean Glaucoma Society graded all the glaucoma-related diseases. All the fundus photography, visual field tests and OCT results were read twice by an independent reader. If there was a disagreement in the primary diagnosis, an independent senior reading committee from Korean Glaucoma Society made the final diagnosis of the glaucoma diseases.

\section{Conclusion}

In this report, we provided the purpose and method for the seventh and eighth KHANES ophthalmic survey. In addition to updating previous KHANES ophthalmic results, this survey had two important aspects that should be 
mentioned. First, this survey will be the world's first nationwide population-based study to use measurements including IOL master and OCT. This will provide objective and the most accurate information regarding ocular structures, including the retina and choroid, and diagnosis of glaucoma and macular diseases, including AMD and DR. Second, in this survey, the whole process, including survey conduction, data collection, and data analysis, are based on an online-based platform. This will make the survey processes more efficient and as objective as possible. In addition, this experience will be used to guide future studies that will use more algorithm-based data processing. Further clinical implications and reports regarding the survey are scheduled for the future.

\section{Conflict of Interest}

No potential conflict of interest relevant to this article was reported.

\section{Acknowledgements}

This work was supported by the Research Program funded by the KDCA (2019E340100).

We are grateful for the support of the KDCA, which provide endless supports to perform the Korea National Health and Nutrition Examination Survey, and members of the Korean Retina Society and Korean Glaucoma Society who participated in the data reading committee.

\section{References}

1. Kweon S, Kim Y, Jang MJ, et al. Data resource profile: the Korea National Health and Nutrition Examination Survey (KNHANES). Int J Epidemiol 2014;43:69-77.

2. Kim HA, Lee SY, Kwon HS, et al. Gender differences in the association of insulin resistance with metabolic risk factors among Korean adolescents: Korea National Health and Nutrition Examination Survey 2008-2010. Diabetes Res Clin Pract 2013;99:54-62.

3. Baek SJ, Nam GE, Han KD, et al. Sarcopenia and sarcopenic obesity and their association with dyslipidemia in Ko- rean elderly men: the 2008-2010 Korea National Health and Nutrition Examination Survey. J Endocrinol Invest 2014;37:247-60.

4. Park TH, Ko Y, Lee SJ, et al. Identifying target risk factors using population attributable risks of ischemic stroke by age and sex. J Stroke 2015;17:302-11.

5. Chon SJ, Choi YR, Roh YH, et al. Association between levels of serum ferritin and bone mineral density in Korean premenopausal and postmenopausal women: KNHANES 2008-2010. PLoS One 2014;9:e114972.

6. Yoon KC, Choi W, Lee HS, et al. An Overview of ophthalmologic survey methodology in the 2008-2015 Korean National Health and Nutrition Examination Surveys. Korean J Ophthalmol 2015;29:359-67.

7. Yoon KC, Mun GH, Kim SD, et al. Prevalence of eye diseases in South Korea: data from the Korea National Health and Nutrition Examination Survey 2008-2009. Korean J Ophthalmol 2011;25:421-33.

8. Park SH, Lee JS, Heo H, et al. A nationwide population-based study of low vision and blindness in South Korea. Invest Ophthalmol Vis Sci 2014;56:484-93.

9. Cho GE, Lim DH, Baek M, et al. Visual impairment of Korean population: prevalence and impact on mental health. Invest Ophthalmol Vis Sci 2015;56:4375-81.

10. Park SJ, Lee JH, Woo SJ, et al. Five heavy metallic elements and age-related macular degeneration: Korean National Health and Nutrition Examination Survey, 20082011. Ophthalmology 2015;122:129-37.

11. Park Y, Shin JA, Yang SW, et al. The relationship between visual impairment and health-related quality of life in Korean adults: the Korea National Health and Nutrition Examination Survey (2008-2012). PLoS One 2015;10:e132779.

12. Rim TH, Nam JS, Choi M, et al. Prevalence and risk factors of visual impairment and blindness in Korea: the Fourth Korea National Health and Nutrition Examination Survey in 2008-2010. Acta Ophthalmol 2014;92:e317-25.

13. Paik JS, Jung SK, Han KD, et al. Obesity as a potential risk factor for blepharoptosis: the Korea National Health and Nutrition Examination Survey 2008-2010. PLoS One 2015;10:e0131427.

14. Park HY, Shin HY, Yoon JY, et al. Intereye comparison of cirrus OCT in early glaucoma diagnosis and detecting photographic retinal nerve fiber layer abnormalities. Invest Ophthalmol Vis Sci 2014;56:1733-42.

15. Medeiros FA, Zangwill LM, Bowd C, et al. The structure 
and function relationship in glaucoma: implications for detection of progression and measurement of rates of change. Invest Ophthalmol Vis Sci 2012;53:6939-46.

16. Bird AC, Bressler NM, Bressler SB, et al. An international classification and grading system for age-related maculopathy and age-related macular degeneration. The International ARM Epidemiological Study Group. Surv Ophthalmol 1995;39:367-74.

17. Grading diabetic retinopathy from stereoscopic color fundus photographs: an extension of the modified Airlie House classification. ETDRS report number 10. Early Treatment Diabetic Retinopathy Study Research Group. Ophthalmology 1991;98(5 Suppl):786-806.

18. Kim KE, Kim MJ, Park KH, et al. Prevalence, awareness, and risk factors of primary open-angle glaucoma: Korea National Health and Nutrition Examination Survey 20082011. Ophthalmology 2016;123:532-41.

19. Kim CS, Seong GJ, Lee NH, et al. Prevalence of primary open-angle glaucoma in central South Korea the Namil study. Ophthalmology 2011;118:1024-30. 